\title{
Obesity and pain
}

British Journal of Pain

7(4) 163-170

(c) The British Pain Society 2013

Reprints and permissions: sagepub.co.uk/

journalsPermissions.nav

DOI: $10.1177 / 2049463713484296$

bjp.sagepub.com

(S) SAGE

\begin{abstract}
Recent reports suggest that almost half of the UK population is expected to be obese by 2030. A number of associations between obesity and chronic pain have been displayed in previous studies, and therefore it can be expected that the presentation of obese patients with chronic pain will rise in accordance with the prevalence of obesity. No single causative relationship between the two can be suggested, so the link between them is believed to be multifactorial. Despite this, little work has been done to draw together the many associations between obesity and pain. A better understanding of their connection, then, is required to appropriately manage this patient group that is increasing in numbers. This article aims to draw together existing evidence on the associations between obesity and pain to create a model of their multifactorial relationship. The article will systematically address each of the current theories in order to gradually build a diagrammatic representation of the relationship, which shall conclude the article.
\end{abstract}

\section{Keywords}

Chronic pain, low back pain, myofascial pain syndromes, pain, somatoform disorders

\section{Introduction}

A number of studies have positively correlated the experience of pain with an increase in body mass index (BMI). ${ }^{1,2}$ With almost $50 \%$ of the UK population projected to be obese by 2030 , costs to the UK economy generated by lost working time of those in chronic pain can be expected to rise. An understanding of the relationship between obesity and pain is crucial to effectively interject the cycle. As yet, the causal relationship between the two remains unclear: it is not known whether obesity causes chronic pain, chronic pain causes obesity or some other factor causes both concurrently. Obesity is hypothesised to lead to pain because of excess mechanical stresses and its proinflammatory state. Chronic pain may result in obesity because of physical inactivity and utilisation of eating for analgesic effect. Genetic, psychological or metabolic factors may also lead to both obesity and pain. ${ }^{2,3}$ This article aims to systematically address each of these hypotheses, investigating their roles in the relationship between obesity and pain, before concluding by combining the hypotheses to create a multifactorial model of this complex relationship.

\section{Results: obesity can cause pain}

\section{Proinflammatory state of obesity}

Obese patients are known to exhibit higher levels of the inflammatory markers interleukin 6 (IL-6), tumour necrosis factor $\alpha(\mathrm{TNF}-\alpha)$ and C-reactive protein (CRP). ${ }^{4,5}$ Adipocytes, as well as providing an energy store for the body, also have an endocrine function, secreting adipocytokines such as IL- $6, \mathrm{TNF}-\alpha$ and leptin. CRP is released from the liver in response to IL-6 among other inflammatory cytokines, normally acting to assist the immune system by activating the complement cascade and opsonising pathogens. IL- 6 has roles that may contribute further to pain, forming the basis of other hypotheses discussed later in this essay. Another adipocytokine released is monocyte chemoattractant protein 1 (MCP-1), which attracts monocytes to the adipose tissue to stimulate them to release their own inflammatory cytokines, including yet more IL-6

\section{Corresponding author:}

Donald Scott McVinnie, Medical Student, University of Glasgow, Glasgow, UK.

Email: 0209073mastudent.gla.ac.uk 


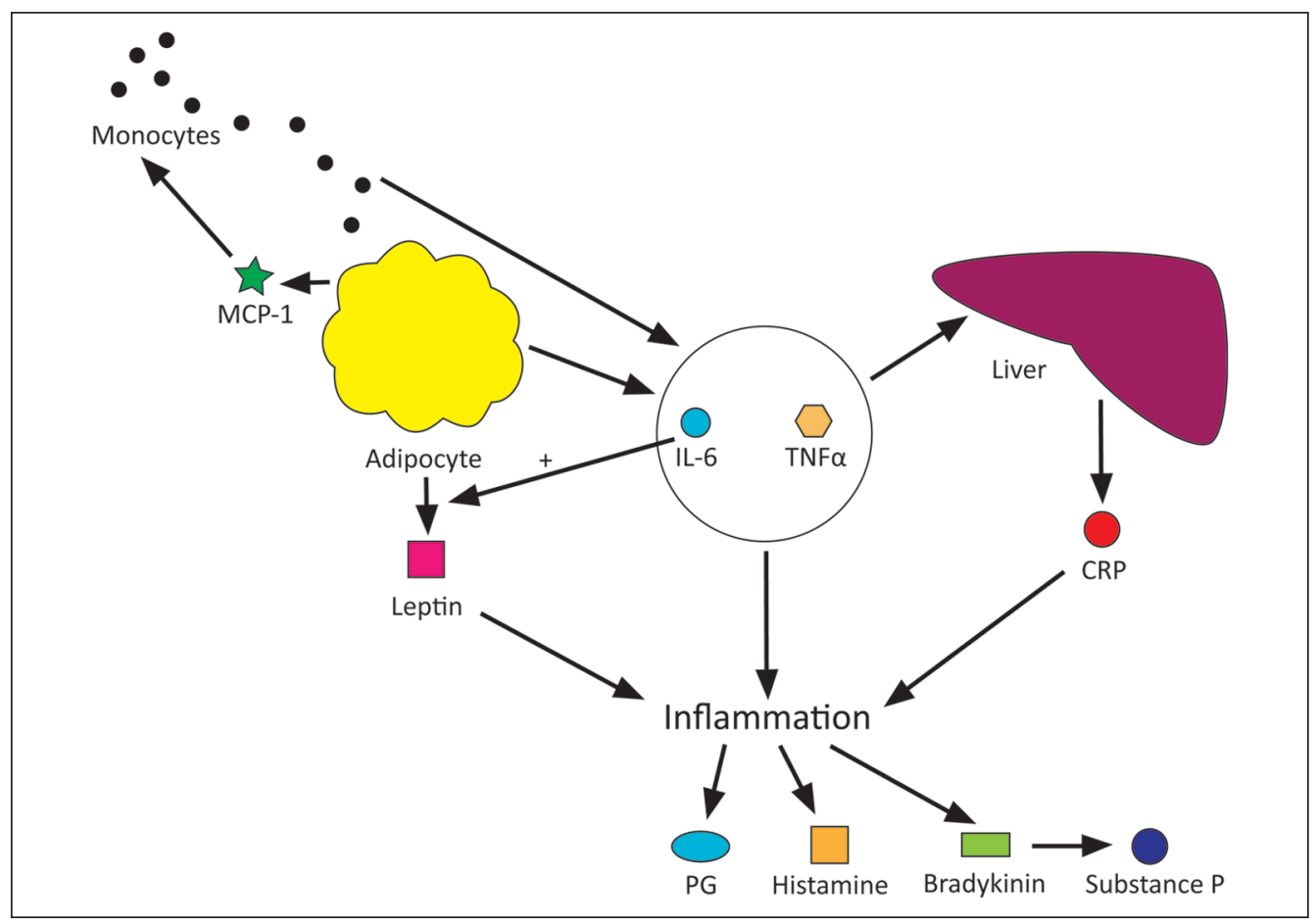

Figure 1. Diagram of obesity as a proinflammatory disorder causing pain.

and TNF- $\alpha$, so amplifying the response. This relationship is depicted in Figure 1.

Watkins et al. ${ }^{6}$ summarise the large body of literature that supports the role of proinflammatory cytokines in producing a hyperalgesic state. An increased quantity of adipose tissue in an individual may then lead to an increased inflammatory response with the chemical mediators involved in inflammation, such as prostaglandins, kinins and histamine, interacting with the nervous system to create a sensation of pain. ${ }^{7}$

It must be noted, however, that while there exists much evidence to support an increased quantity of inflammatory cytokines in obese individuals and much theory in how inflammatory cytokines contribute to pain, there is as yet very little evidence to directly link obesity with pain through inflammatory cytokine production, and this perhaps is an area for future study.

\section{Mechanical stresses}

Increased mechanical stresses on the body in obesity are believed to result in an increased risk of musculoskeletal and joint pain. A number of studies have shown a positive correlation between increased BMI and increased musculoskeletal pain, particularly of low back pain and lower limb pain. ${ }^{8-10}$ The hypothesis behind this link is that overloading the lower back, hip and knee joints causes injury and degradation to these structures, leading to osteoarthritis. Mechanoreceptors on chondrocytes are activated by the mechanical stimulation of overloading on joints leading to the activation of intracellular pathways that result in the production of metalloproteases and interleukin 1 (IL1). Metalloproteases degrade cartilage extracellular matrix while IL-1 activates proinflammatory prostaglandins and cytokines (Figure 2).

Mechanoreceptors on bone have a similar effect on overload stimulation, ${ }^{11}$ also resulting in degradation of bone matrix. ${ }^{12}$ It therefore seems logical that with increased BMI these mechanoreceptors may be stimulated more frequently, leading to degeneration of cartilage and bone with associated inflammation. It is interesting to note that there is also a positive correlation between increasing BMI and upper limb pain, but it could not be accounted for by this hypothesis. ${ }^{13}$

\section{Results: factors contributing to both obesity and pain}

\section{Metabolic syndrome}

Metabolic syndrome is the name attributed to a group of risk factors that together increase the risk of cardiovascular disease and stroke. In accordance with the World Health Organization's criteria, ${ }^{14}$ the metabolic syndrome requires diabetes mellitus, impaired glucose tolerance or impaired fasting glucose, as well as two or more of either hypertension, dyslipidaemia, central obesity or microalbuminuria. 


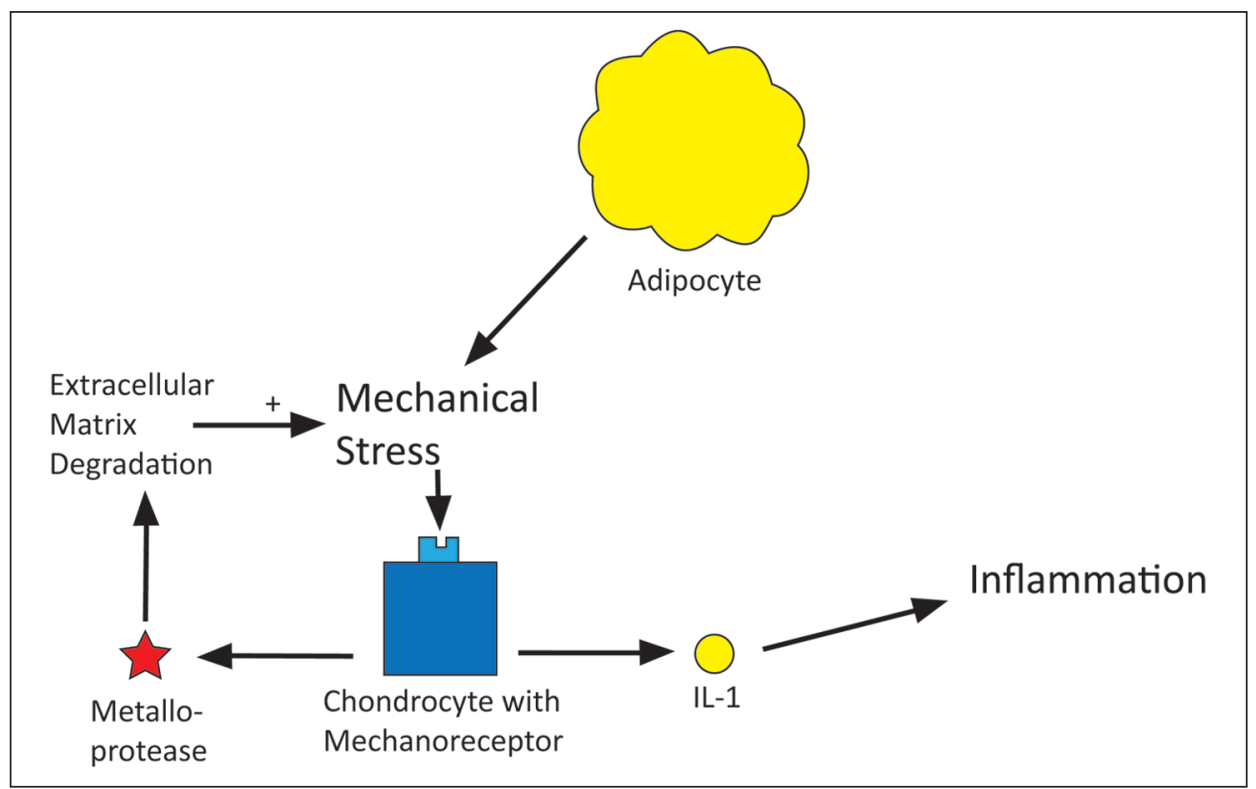

Figure 2. Diagram of obesity causing mechanical stresses which cause pain.

Prevalence of metabolic syndrome increases with increasing BMI and with chronic pain, ${ }^{3,15,16}$ and so it may be that some confounding factor of metabolic syndrome relates obesity to pain. This is fathomable given that, for example, diabetes is already known to be associated with neuropathy. Neuroendocrine levels may provide the link between metabolic syndrome and pain. Hyperinsulinaemia, as occurs early in insulin resistance, can stimulate the sympathetic nervous system with release of noradrenaline. Sympathetic catecholamines have been shown to interact with afferent fibres in pathological conditions with some pain-sensitive nerve fibres expressing $\alpha 2$-adrenoceptors. ${ }^{17}$ In support of this hypothesis one study showed that female fibromyalgia sufferers exhibited higher levels of noradrenaline. ${ }^{15}$ Conversely, one study was noted, which postulated that chronic pain may actually cause metabolic syndrome with persistent psychological stress resulting in raised catecholamine and cortisol levels. ${ }^{16}$ Also, inflammatory cytokines, notably IL-1, are noted to cause an increase in cortisol levels through stimulation of the hypothalamic-pituitary-adrenal axis. The persistently high cortisol levels caused by psychological stress and inflammatory induction counteract insulin's action to raise blood glucose levels and may result in insulin resistance. This is illustrated in Figure 3.

There is, however, little supportive research on links between the metabolic syndrome, obesity and pain. One study, which adjusted results linking obesity and pain for all markers of metabolic syndrome, found that central obesity was the only significant correlation between the two and that irregularities in insulin levels, lipid levels and blood pressure were not associated with pain. ${ }^{3}$

\section{Depression}

Several studies have investigated the relationship between pain and depression, showing a clear link between the two. ${ }^{18-20}$ Some experimental evidence also exists linking obesity to depression, although it is noted that clinical experience more than experimental evidence consistently supports this link. ${ }^{19,21}$ Two of the above-noted studies indicate that depression and obesity are the major risk factors in the incidence of pain. ${ }^{18,21}$ Pain, obesity and depression appear to share a complex reciprocal relationship. Inflammation has been hypothesised as having a central role in this pathophysiological relationship. IL-6, produced by adipocytes (as previously noted), has been correlated to depressive symptoms in patients in previous studies. ${ }^{22}$ IL-6 is alleged to activate the enzyme indoleamine 2,3-dioxygenase, an enzyme which competes with tryptophan hydroxylase for tryptophan. The significance of this is that serotonin, which is normally produced from tryptophan by action of tryptophan hydroxylase, becomes less available because of the activation of the competing pathway. ${ }^{23}$ Serotonin depletion is well known to be involved in the pathophysiology of depression, as can be seen through the success of selective serotonin reuptake inhibitors in the treatment of depression. Serotonin is also implicated in the descending modulation of pain. Reduced levels of serotonin may result not only in depression but also in reduced effectiveness of the descending modulatory 


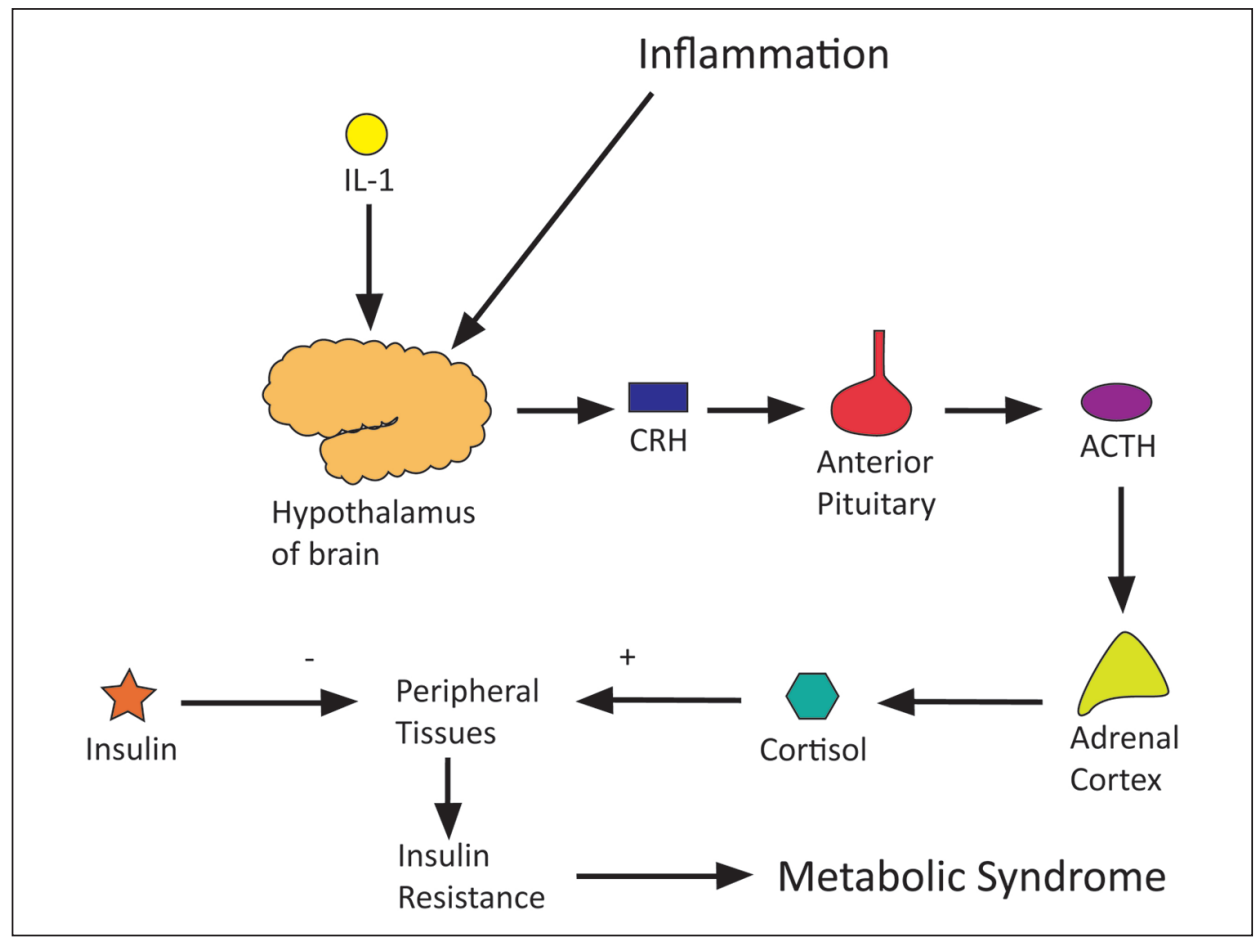

Figure 3. Diagram of how chronic pain and obesity cause the metabolic syndrome.

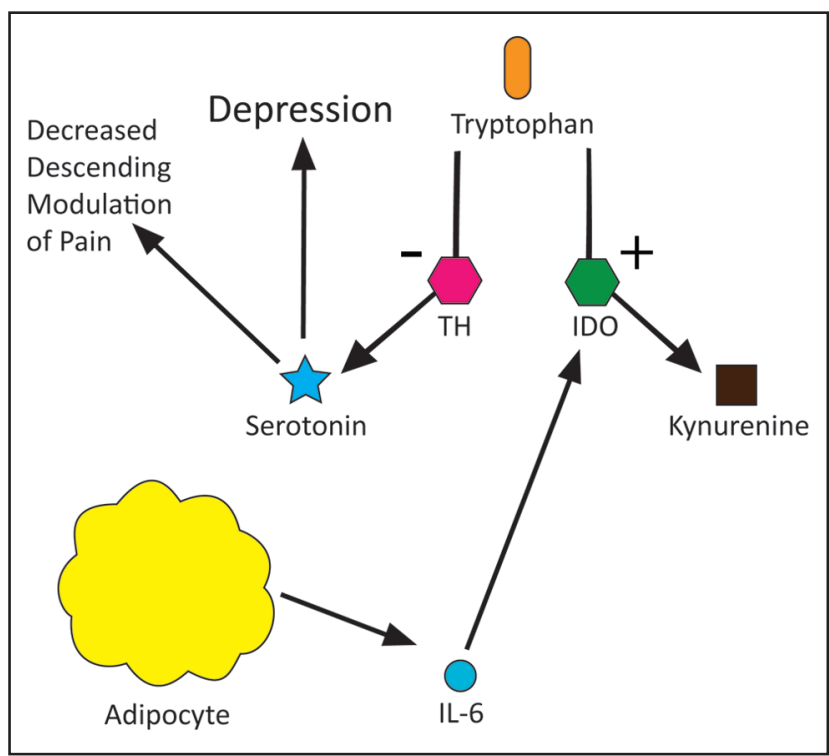

Figure 4. Diagram of how depression, obesity and pain interact.

pathway, thereby increasing the magnitude of pain experienced by an individual. ${ }^{24}$ These relationships are illustrated in Figure 4.

\section{Genetics}

Relatively few studies have examined the possible genetic links between obesity and pain. Two separate studies examining these links in twins have been carried out. One study, which examined the relationship between obesity and pain, adjusted for genetic factors and factors that resulted from twins being brought up in a shared environment. The results of this study showed that some part of the relationship between obesity and pain can be explained by either genetic factors or familial shared environmental factors; however, the study did not differentiate between these two factors. ${ }^{25}$ Results of another study, which examined monozygotic twins with differing BMIs, showed that pain was remarkably similar in each twin within a pair despite sometimes large differences in $\mathrm{BMI}$, again suggesting a possible genetic cause of their pain. ${ }^{26}$ It is not difficult to accept a genetic link between obesity and pain given the understanding that obesity itself is highly hereditable with genetics suggested to account for $50-80 \%$ of the risk of obesity. ${ }^{26}$ Unipolar depression also shows hereditability, exhibiting a concordance of $30-60 \%$ in monozygotic twins. ${ }^{27}$

\section{Results: pain can cause obesity}

\section{Sedentary lifestyle}

Chronic pain may lead to obesity in previously nonobese individuals. One reason for this subsequent weight gain is adoption of a sedentary lifestyle. A study of the impact of knee osteoarthritis on physical activity found that only $12.9 \%$ of male and $7.7 \%$ of female 
patients with knee osteoarthritis were meeting the recommended weekly levels of exercise. ${ }^{28}$ Another study positively correlated level and duration of pain with resultant weight gain; those individuals with the longest-lasting chronic low back pain experienced greater gains in weight than asymptomatic individuals. ${ }^{29}$ These studies suggest that obesity is not necessarily completely causal of pain, but may be secondary to lifestyle adaptations associated with pain.

A cognitive-behaviourist hypothesis suggests that the progression to an inactive sedentary lifestyle is experienced by some patients in chronic pain. A threestage model, constructed by Gatchel in 1991, was constructed to track the progression from acute pain to chronic pain and the input of cognitive processes (Table 1)..$^{30}$

Table 1. Three-stage model of progression from acute to chronic pain and input of cognitive processes by Gatchel ${ }^{31}$.

\begin{tabular}{ll}
\hline Stage & Reaction \\
\hline 1 & $\begin{array}{l}\text { Normal emotional reactions (e.g. fear, anxiety) } \\
\text { resulting from acute pain perception }\end{array}$ \\
2 & $\begin{array}{l}\text { Behavioural reactions (e.g. learned helplessness, } \\
\text { distress) from chronic pain lasting 2-4 months as } \\
\text { may occur with inappropriate attention }\end{array}$ \\
3 & $\begin{array}{l}\text { Acceptance or habituation of the 'sick role' } \\
\text { excusing the individual from his or her normal } \\
\text { daily roles, which acts to positively reinforce this } \\
\text { behaviour }\end{array}$ \\
\hline
\end{tabular}

According to this model, the patient adopts operant-learned avoidance behaviours as a result of anxiety due to the expectation that activity exacerbates pain. This lack of activity results in less pain being experienced but also in physical deconditioning, actually increasing the likelihood of pain. This lack of mobilisation then contributes to obesity. ${ }^{2}$ It is noted that entry to stage two of Gatchel's model may be predisposed by certain personality types and avoided by others.

\section{Eating analgesia}

Research indicates that eating pleasurable foods, socalled 'hedonic eating', ${ }^{32}$ confers analgesic effect. Experimental rat models have shown that painful stimuli applied to subjects that are eating pleasurable foods produces a delayed response in comparison with control subjects. ${ }^{32}$ In these studies the pleasure derived from the food was the most important factor in the analgesic response, with ingestion of unpleasant foods associated with no increased analgesic effect. Interestingly, no relationship was found between the calorific content and degree of analgesic response.

Stimulation of the descending modulatory pathway from the periaqueductal grey is suggested to be integral to this response, as is an area of the brain named the raphe magnus. Furthermore, endogenous opioids are implicated in the response as exhibited by a loss of analgesic effect on inhibition of endogenous opioids. ${ }^{33}$ It is suggested that pleasurable eating stimulates these components in order to inhibit the sensation of pain. ${ }^{32}$

This analgesic effect was found to take place only during consumption of the foodstuff, not prior to or after consumption, and so it follows that for continued analgesic effect one would theoretically have to continue eating. Excessive eating may then be positively reinforced in individuals who experience chronic pain due to this analgesic effect. While the above-mentioned studies noted no requirement of calorific content of food to stimulate an analgesic effect, the simple availability of pleasurable caloriedense food to humans makes excess calorie intake more likely.

Previous work by Mercer and Holder shows that, while these experiments above were carried out on rat models, there is some evidence to suggest similar mechanisms in humans. ${ }^{34}$

\section{Conclusion}

Obesity and pain have a complex interwoven relationship, and it appears that each of the hypotheses discussed in this article to explain this relationship are interrelated. Obesity is recognised as being a proinflammatory state, the subsequent inflammation causing pain to the individual. Inflammatory cytokines cause chronically raised levels of circulating cortisol, which may eventually lead to insulin resistance and, consequently, metabolic syndrome. The increased mechanical stresses of obesity, particularly on the lower limbs, cause chondrocytes to produce proteins which break down cartilage matrix as well as yet more proinflammatory cytokines. The inflammatory cytokines produced in obesity have been shown to shift the balance of serotonin/kynurenine production from tryptophan in favour of kynurenine, the reduced serotonin levels contributing to depression and increased pain experience. Both depression and pain contribute to avoidance behaviour and adoption of a sedentary lifestyle, further precipitated by joint immobility resulting from cartilage degradation. Although a sedentary lifestyle appears to reduce pain exacerbation, it actually contributes to physical deconditioning and obesity, which results in a greater pain experience for the individual. Furthermore, eating is used both as a coping 


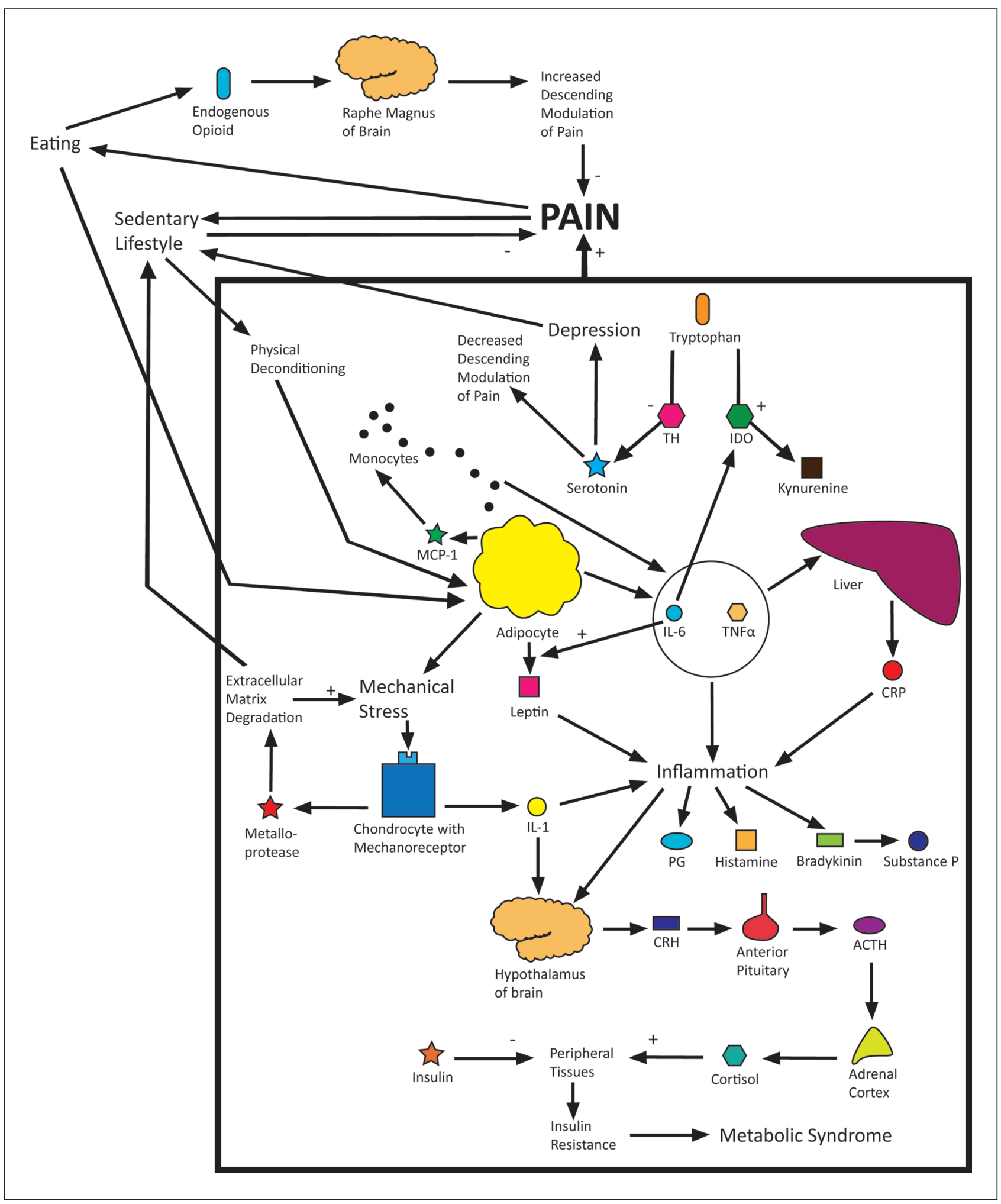

Figure 5. Model of obesity and pain.

mechanism and as a form of analgesia, which temporarily reduces pain and depression but leads again to further weight gain and greater pain experience. Genetics may predispose an individual to entering this cycle at any number of areas mentioned above. A full model has been drawn to incorporate all of the above hypotheses (Figure 5) to illustrate the complex relationship of obesity and pain. Treatment of obese 
patients with chronic pain must take into account this complex relationship.

\section{Acknowledgements}

I would like to express great thanks to Dr Mick Serpell for his much appreciated time and suggestions in compiling this report.

\section{Funding}

This research received no specific grant from any funding agency in the public, commercial, or not-for-profit sectors.

\section{References}

1. Hitt H, McMillen RC, Thornton-Neaves T, Koch K and Cosby AG. Comorbidity of obesity and pain in a general population: results from the Southern Pain Prevalence Study. F Pain 2007; 8(5): 430-436.

2. Somers TJ, Wren AA and Keefe FJ. Understanding chronic pain in older adults: abdominal fat is where it is at. Pain 2011; 152(1): 8-9.

3. Ray L, Lipton RB, Zimmerman ME, Katz MJ and Derby CA. Mechanisms of association between obesity and chronic pain in the elderly. Pain 2011; 152(1): 53-59.

4. Das UN. Is obesity an inflammatory condition? Nutrition 2001; 17(11-12): 953-966.

5. Okifuji A, Donaldson GW, Barck L and Fine PG. Relationship between fibromyalgia and obesity in pain, function, mood, and sleep. F Pain 2010; 11(12): 1329-1337.

6. Watkins LR, Maier SF and Goehler LE. Immune activation: the role of pro-inflammatory cytokines in inflammation, illness responses and pathological pain states. Pain 1995; 63(3): 289-302.

7. Dray A. Inflammatory mediators of pain. Br $\mathcal{F}$ Anaesth 1995; 75: 125-131.

8. Peltonen M, Lindroos AK and Torgerson JS. Musculoskeletal pain in the obese: a comparison with a general population and long-term changes after conventional and surgical obesity treatment. Pain 2003; 104(3): 549-557.

9. Blagojevic M, Jinks C, Jeffery A and Jordan KP. Risk factors for onset of osteoarthritis of the knee in older adults: a systematic review and meta-analysis. Osteoarthr Cartilage 2010; 18(1): 24-33.

10. Christensen R, Bartels EM, Astrup A and Bliddal H. Effect of weight reduction in obese patients diagnosed with knee osteoarthritis: a systematic review and metaanalysis. Ann Rheum Dis 2007; 66: 433-439.

11. Berenbaum F and Sellam J. Obesity and osteoarthritis: what are the links? Foint Bone Spine 2008; 75(6): 667-668.

12. Gabay O, Hall DJ, Berenbaum F, Henrotin Y and Sanchez C. Osteoarthritis and obesity: experimental models. Foint Bone Spine 2008; 75(6): 675-679.

13. Oliveria SA, Felson DT, Cirillo PA, Reed JI and Walker AM. Body weight, body mass index, and incident symptomatic osteoarthritis of the hand, hip and knee. Epidemiology 1999; 10(2): 161-166.
14. World Health Organization. Definition, diagnosis and classification of diabetes mellitus and its complications. Geneva: WHO, 1999

15. Loevinger BL, Muller D, Alonso C and Coe CL. Metabolic syndrome in women with chronic pain. Metabolism 2007; 56(1): 87-93.

16. Rezania K, Soliven B, Rezai KA and Roos RP. Impaired glucose tolerance and metabolic syndrome in idiopathic polyneuropathy: the role of pain and depression. Med Hypotheses 2011; 76(4): 538-542.

17. Martinez-Lavin M. Fibromyalgia as a sympathetically maintained pain syndrome. Curr Pain Headache Rep 8(5): 385-389.

18. ShiY, Hooten WM, Roberts RO and Warner DO. Modifiable risk factors for incidence of pain in older adults. Pain 2010; 151(2): 366-371.

19. Ohayon MM and Schatzberg AF. Chronic pain and major depressive disorder in the general population. $\mathcal{F}$ Psychiatr Res 2010; 44(7): 454-461.

20. Kopec JA. Predictors of back pain in a general population cohort. Spine 2004; 29(1): 70-77.

21. Hill AJ. Psychological aspects of obesity. Psychiatry 2005; 4(4): 26-30.

22. Miller GE, Freedland KE, Duntley S and Carney RM. Relation of depressive symptoms to C-reactive protein and pathogen burden (cytomegalovirus, herpes simplex virus, Epstein-Barr virus) in patients with earlier acute coronary syndromes. Am f Cardiol 2005; 95(3): 317-321.

23. Shelton RC and Miller AH. Eating ourselves to death (and despair): the contribution of adiposity and inflammation to depression. Prog Neurobiol 2010; 91(4): 275-299.

24. Kroenke K, Wu J, Bair MJ, Krebs EE, Damush TM and Tu W. Reciprocal relationship between pain and depression: a 12-month longitudinal analysis in primary care. f Pain 2011; 12(9): 964-973.

25. Wright LJ, Schur E, Noonan C, Ahumada S, Buchwald $\mathrm{D}$ and Afari N. Chronic pain, overweight, and obesity: findings from a community-based twin registry. $\mathcal{F}$ Pain 2010; 11(7): 628-635.

26. Leboeuf-Yde CDC, Kyvik KO and Bruun NH. Low back pain and lifestyle. Part II - obesity: information from a population-based sample of 29,424 twin subjects. Spine 1999; 24(8): 779-784.

27. Kumar P and Clark M. Kumar E Clark's clinical medicine. 7th ed. Edinburgh: Saunders Elsevier, 2009.

28. Lee R and Kean WF. Obesity and knee osteoarthritis. Inflammopharmacology 2012; 20(2): 53-58.

29. Lake JK, Power C and Cole TJ. Back pain and obesity in the 1958 British birth cohort: cause or effect? F Clin Epidemiol 2000; 53(3): 245-250.

30. Gatchel RJ. Early development of physical and mental deconditioning in painful spinal disorders. In: Mayer TG, Mooney V and Gatchel RJ eds. Contemporary conservative care for painful spinal disorders. Philadelphia, PA: Lea \& Febiger, 1991.

31. Dersh J, Polatin PB and Gatchel RJ. Chronic pain and psychopathology: research findings and theoretical considerations. Psychosom Med 2002; 64(5): 773-786. 
32. Foo $\mathrm{H}$ and Mason P. Analgesia accompanying food consumption requires ingestion of hedonic food. $\mathcal{F}$ Neurosci 2009; 29(41): 13053-13062.

33. de Freitas RL, Kübler JML, Elias-Filho DH and Coimbra NC. Antinociception induced by acute oral administration of sweet substance in young and adult rodents: the role of endogenous opioid peptides chemical mediators and $\mu 1$-opioid receptors. Pharmacol Biochem Behav 2012; 101(2): 265-270.

34. Mercer ME and Holder MD. Antinociceptive effects of palatable sweet ingesta on human responsivity to pressure pain. Physiol Behav 1997; 61(2): 311-318. 